\title{
Representing SimModel in the Web Ontology Language
}

\author{
P. Pauwels ${ }^{1}$, E. Corry ${ }^{2}$ and J. O’Donnell ${ }^{3}$ \\ ${ }^{1}$ Department of Architecture and Urban Planning, Ghent University, J. Plateaustraat \\ 22, 9000 Ghent, Belgium; email: pipauwel.pauwels@ugent.be \\ ${ }^{2}$ Informatics Research Unit for Sustainable Engineering, National University Ireland \\ Galway, Galway, Ireland; email: edward.corry@nuigalway.ie \\ ${ }^{3}$ University College Dublin, Dublin, Ireland; email: james.odonnell@ucd.ie
}

\begin{abstract}
Many building energy performance (BEP) simulation tools, such as EnergyPlus and DOE-2, use custom schema definitions (IDD and BDL respectively) as opposed to standardised schema definitions (defined in XSD, EXPRESS, and so forth). A Simulation Domain Model (SimModel) was therefore proposed earlier, representative for a new interoperable XML-based data model for the building simulation domain. Its ontology aims at moving away from tool-specific, nonstandard nomenclature by implementing an industry-validated terminology aligned with the Industry Foundation Classes (IFC). In this paper, we document our ongoing efforts to make building simulation data more interoperable with other building data.

In order to be able to better integrate SimModel information with other building information, we have aimed at representing this information in the Resource Description Framework (RDF). A conversion service has been built that is able to parse the SimModel ontology in the form of XSD schemas and output a SimModel ontology in OWL. In this article, we document this effort and give an indication of what the resulting SimModel ontology in OWL can be used for.
\end{abstract}

\section{SHARING INFORMATION IN CONSTRUCTION INDUSTRY}

Projects in the domain of architecture, engineering and construction (AEC) typically involve diverse parties, each bringing specific information into these projects. Client information needs to be combined with the information of the architectural design firm; electrical engineering information needs to be combined with facility management information; plumbing information needs to be combined with sensor information; and so forth. Also after the construction phase, building information needs to be accessible for a range of diverse users, including the facility director, inhouse machinery and systems, renovation specialists, technicians, and so forth. As a result, a well-functioning information flow throughout the complete Building LifeCycle (BLC) is crucial.

This is also the case for the information flow within one of the subdomains of the AEC domain, namely the domain of building energy performance (BEP) simulation (Bazjanac, 2004; O'Donnell et al., 2013). In this article, we will therefore look into one of the diverse options to make information for BEP simulation increasingly interoperable (Bazjanac et al., 2011a). 
Various strategies exist for sharing (building) information within the AEC domain for enabling an improved level of interoperability. Some of these strategies have been outlined in (Pauwels et al., 2010, 2011) and (Törmä, 2013). In short, a distinction can be made between the three following approaches.

1. Comparison / sharing in the wild

2. Fusion / centralised model

3. Linking / linked building data

In the strategy of sharing in the wild all partners in a building project share information as they see fit (time, format, means of communication, and so forth - see Fig. 1 left). This is the most traditional and most common strategy for exchanging information in the AEC domain. In this case, the diverse people that manage (their part of) all the information are of crucial information as they are supposed to interpret incoming information and translate / convert it manually to their own model of the building project. As has been shown by, this is a time-consuming and errorprone strategy (Bazjanac, 2001).
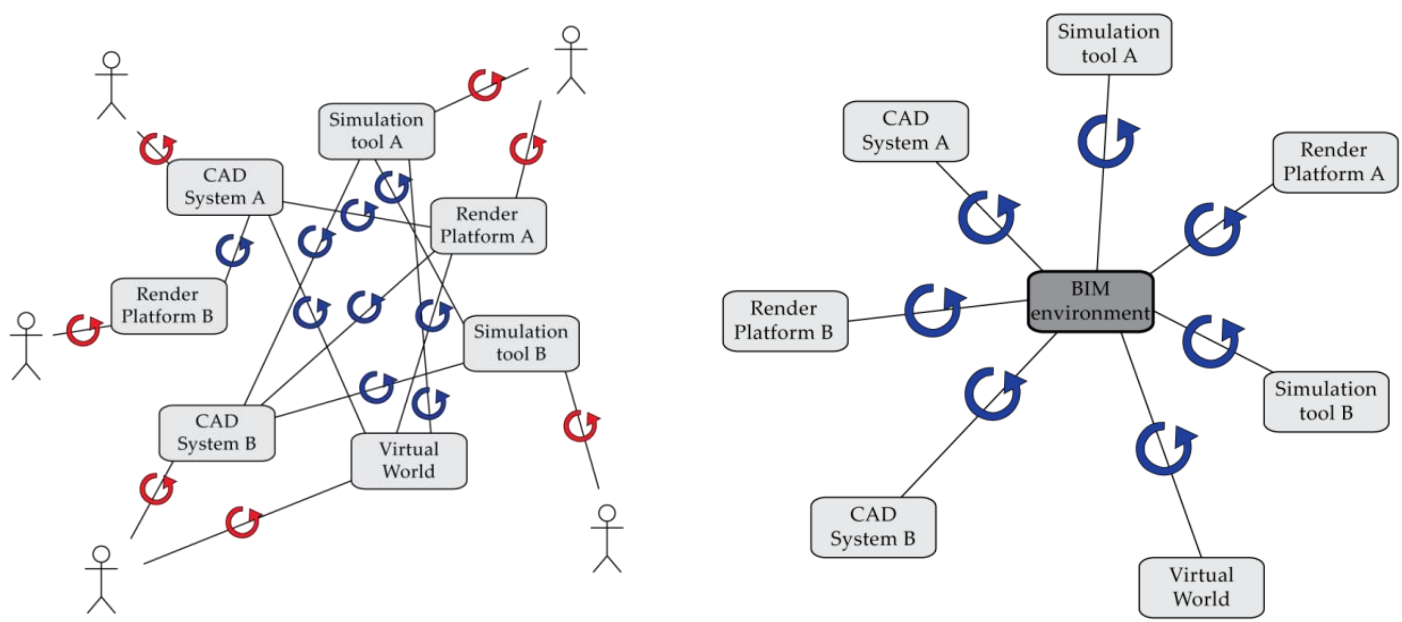

Figure 1: When sharing information 'in the wild' (left), end users need to manually compare new information with information that they manage. In a centralised model strategy (right), on the other hand, information is stored in one central location, and other applications refer to that information only and store to that central model only.

In a centralised model strategy, information is gathered in one location and preferably in one (consistent) model (Fig. 1 right). Exemplar to such a strategy is the building information modelling (BIM) approach (Eastman et al., 2011). In this case, one central BIM model is put at the heart of a building project and every partner is required to make changes and update that central model. This approach is further enhanced by a standard file format for representing and sharing building information, namely the Industry Foundation Classes (IFC) of the BuildingSMART alliance (buildingSMART, 2013; Liebich et al., 2009). Also the centralised model approach is 
problematic, since projects in the AEC domain prove to be too diverse to enable a well-functioning information exchange among all partners in this centralised model strategy. It often happens that information does not fit into the central model, whether this is through the IFC file format or any other industrial standard. In the case of IFC, standardised information exchange requirements called Model View Definitions are viewed as the solution to this issue (buildingSMART, 2010).

Finally, in a linked building data approach (Fig. 2), diverse information sources used within a building project are linked together as needed, and a management system is set up on top of those linked information sources for managing the links between the diverse models (Pauwels et al., 2010, 2011). The links made between building models are often pairwise. Also this approach has its issues. The linked building data consists mainly of pairwise links, resulting in loosely connected data resembling more to the 'sharing in the wild' (Fig. 1 left) approach than to a centralised approach. As a result, it is not a straightforward matter to provide a management system and appropriately manage the diverse links in an intuitive manner.

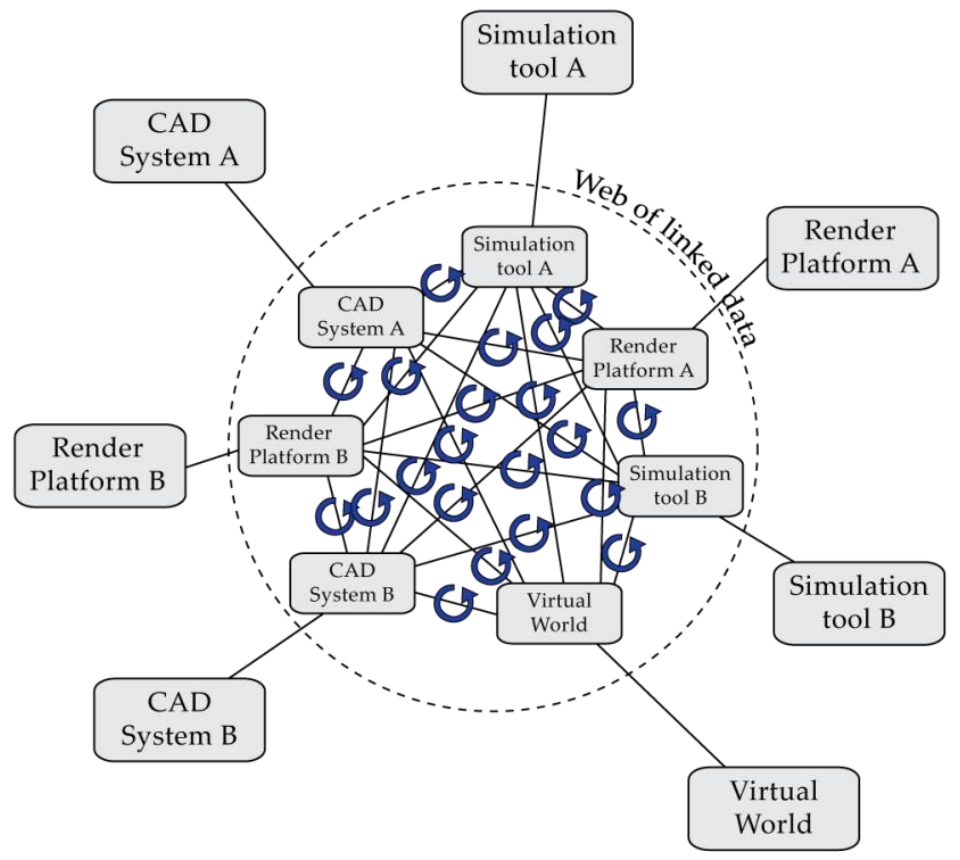

Figure 2: In a linked building data strategy, information keeps following the format that is required by the application that is using it (nodes outside dashed circle). Additionally, it is tightly linked together on a data-level (nodes inside dashed circle), so that information in one format / application can be related to information in another format / application.

\section{LINKED BUILDING DATA}

Of the three above approaches, the last 'linking' approach or 'linked building data' strategy seems promising, although it still needs to prove its value in practice. When considering the domain of BEP simulations, many different models are used in 
parallel, and not often is there one central BEP model. For example, EnergyPlus and DOE-2 represent two such parallel schemas, which are not often used in combination within a project, let alone in a centralised model strategy, in which one of them precedes the other in importance. In (Bazjanac et al., 2011a, 2011b), a SimModel was proposed with the aim of bringing such models (IFC, gbXML, EnergyPlus, DOE-2) together into one centralised schema. It remains to be seen whether or not the SimModel ontology will eventually indeed be used in a centralised manner or not. If it is not, it still makes sense to combine SimModel information with other building models available in the AEC project and with data outside the AEC project (e.g. more static references, such as material information and geographic information). For this purpose, we will test the 'linking' approach documented above (Fig. 2).

At the core of the linked building data approach are semantic web technologies (Berners-Lee et al., 2001). Central is the Resource Description Framework (RDF), which is a data model for the representation of any information in RDF graphs (Manola and Miller, 2004). An RDF graph is a directed labelled graph, following a triple structure for each statement (object - predicate - subject). Using this structure, one can represent near to any information. The Web Ontology Language is based on the RDF data model and allows to represent ontologies (W3C OWL Working Group, 2012).

In the following sections, we give a brief overview of SimModel and how it is converted into an OWL ontology. Once this OWL ontology is available, information can be represented in RDF graphs, following the OWL ontology of SimModel. Semantic web technologies can then be used for handling the represented information and, finally, the linked building data approach of Fig. 2 can be realised for BEP simulation data.

\section{SIMMODEL}

SimModel is primarily used as an internal data model by the Simergy software developed at LBNL (LBNL, 2013; See et al., 2011), the successor of the Mojito platform presented by (Bazjanac et al., 2011). The Mojito tool was first conceived as a platform that facilitates data flow to and from BEP simulation tools to and from potentially any building modelling tool (Bazjanac et al., 2011a). Data flow is possible to and from BIM models in IFC, DOE-2 software or tools that use the DOE2 engine, EnergyPlus, and tools with gbXML export. These tools are typically used for BEP simulations. Data from any of these environments can be mapped to and from the SimModel data model using the Simergy software (LBNL, 2013). SimModel can thus be placed among other AEC domain tools as displayed in Fig. 3.

Simergy is a comprehensive graphical user interface (GUI) for the US Department of Energy's (DOE) building energy simulation program EnergyPlus (LBNL, 2013; O'Donnell et al., 2013; See et al., 2011). The application provides an intuitive schematic editor for HVAC systems. The editor handles on a space-based building 
model, which can be imported from the diverse sources shown in Fig. 3 (O'Donnell et al., 2011).

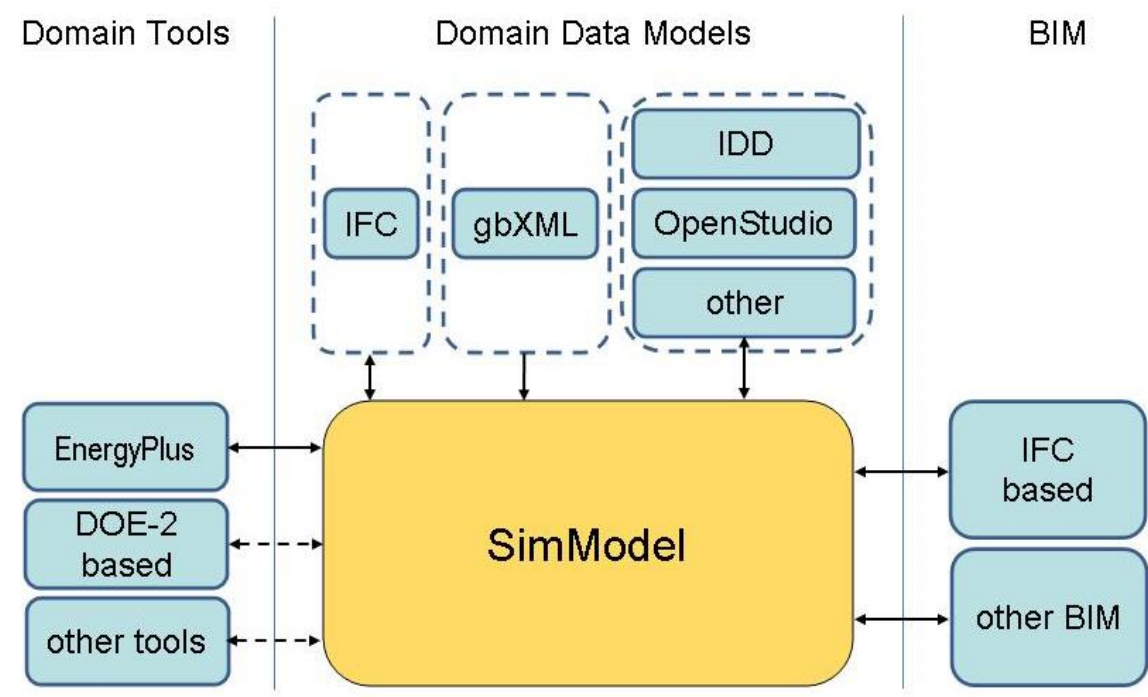

Figure 3: SimModel in relation to existing building energy performance software and models (original image in Bazjanac et al., 2011).

The underlying SimModel is an object-oriented data model which defines all object / attribute / relationship sets used for building energy performance (BEP) simulation. "[T]he primary objective of SimModel is to accommodate the existing input data requirement of EnergyPlus, while allowing mapping from/to other domain data models and easy incorporation of new definitions." (Bazjanac et al., 2011b). At the core, SimModel is represented using the XML markup language (O'Donnell et al., 2011). This representation is closely aligned to the IFC data model, so that it can be linked to incoming or outgoing IFC information (Fig. 3).

An indication of the SimModel hierarchy and structure was documented before (Bazjanac et al., 2011a; O'Donnell et al. 2011). The model has a 'project' node at the top of its hierarchy, which is decomposed by various design alternatives. Each design alternative is then eventually decomposed by a 'building' node, which includes 'building elements', 'zones', 'HVAC systems' and 'other systems'. The nodes 'building elements', 'zones', and 'HVAC systems' hereby map directly to the diverse domain models displayed in Fig. 3. The node 'other systems' is included to enable future extensions of the SimModel (e.g. addition of electrical systems).

\section{CONVERSION TO AN OWL ONTOLOGY}

In our approach to convert the SimModel into an OWL ontology, we started from the XML Schema Definition (XSD) files that represent the SimModel. The five following XSD files are available, each representing a part of the SimModel data model. 
1. SIM core

http://www.lbl.gov/namespaces/Sim/SimModelCore

simcore.xsd

2. SIM building model

http://www.lbl.gov/namespaces/Sim/BuildingModel

simbldg.xsd

3. SIM resources general

http://www.lbl.gov/namespaces/Sim/ResourcesGeneral

simres.xsd

4. SIM resources geometry

http://www.lbl.gov/namespaces/Sim/ResourcesGeometry

simgeom.xsd

5. SIM MEP model

http://www.lbl.gov/namespaces/Sim/MepModel

simmep.xsd

All five XSD files are tightly linked together with cross-references to each other's classes. SimModel contains a few hundred classes and it is hierarchically structured using classes, types and subtypes (O'Donnell et al. 2011). In the XSD files, this results in a hierarchy of xs:complexType elements. A schematic outline is given below for the SimFlowMover class and three of its six subtypes (Default, Fan, Pump, PumpSet, ReturnFan, SupplyFan) in the SIM MEP model of the SimModel.

complexType SimFlowMover

abstract complexType SimFlowMover_Default

complexType SimFlowMover_Default_Default

abstract complexType SimFlowMover_Fan

complexType SimFlowMover_Fan_NightVentilation

complexType SimFlowMover_Fan_ZoneExhaust

abstract complexType SimFlowMover_Pump

complexType SimFlowMover_Pump_ConstantSpeedReturn

complexType SimFlowMover_Pump_ConstantSpeedSupply

complexType SimFlowMover_Pump_UserDefined

complexType SimFlowMover_Pump_VariableSpeedReturn

complexType SimFlowMover_Pump_VariableSpeedSupply

complexType SimFlowMover_Pump_VarSpeedCondensateReturn

complexType SimFlowMover_Pump_VarSpeedCondensateSupply

In our converter application, each of the five XSD files is parsed and converted into a corresponding OWL ontology file, while keeping track of the cross references. Each class or subtype is converted into an OWL class (owl:Class), referring to an upper class when required, as shown below.

simmep:SimFlowMover_Fan

rdfs:subClassOf simmep:SimFlowMover ;

rdf:type owl:Class . 
For each class, the required properties are generated as owl:DatatypeProperty or owl:ObjectProperty declarations (see below), resulting in a complete representation of the SimModel in five ontology files.

simmep:simFlowMover_SimFlowMover_Name

rdf:type owl:DatatypeProperty;

rdf:type owl:FunctionalProperty;

rdfs:domain simmep:SimFlowMover;

rdfs:range xsd:string .

\section{CONCLUSION}

In this article, we documented the origin of the SimModel. We outlined how the SimModel is aimed to combine diverse schema definitions in building energy performance (BEP) simulation tools, such as EnergyPlus, DOE-2, IFC, gbXML, and so forth. It is conceived as an interoperable XML-based data model for the building simulation domain. As such, it can be aligned with many of the existing applications for BEP simulation.

In order to be able to better integrate SimModel information with other building information, we have aimed at representing SimModel information in RDF graphs. A first step towards this aim is presented here in the form of a conversion service of the XML-based SimModel schema into OWL ontologies. With this ontology, one is able to represent SimModel information in well-structured RDF graphs, so that these graphs can be used by more advanced semantic web technologies, such as reasoning engines with a basis in Description Logics (DL) or SPARQL query interfaces. Furthermore, the possibility of representing SimModel information in RDF graphs enables addressing interoperability issues in the AEC domain using a linked building data approach. In this approach, the SimModel information of the BEP simulation domain can be more easily integrated with information outside the BEP simulation, to enable for instance a better overall building lifecycle management.

\section{REFERENCES}

Bazjanac, V. (2001). "Acquisition of building geometry in the simulation of energy performance." 7th International Building Performance Simulation Association (IBPSA) Conference.

Bazjanac, V. (2004). "Building energy performance simulation as part of interoperable software environments." Build. Environ. 39, 879-883.

Bazjanac, V., Maile, T., O’Donnell, J., Rose, C., and Mrazovic, N. (2011a). "Data environments and processing in semi-automated simulation with EnergyPlus." 28th International CIB W078-W102 Conference.

Bazjanac, V., Maile, T., Rose, C., O’Donnell, J., Mrazović, N., Morrissey, E., and Welle, B. (2011b). "An assessment of the use of building energy performance simulation in early design." 12th International Building Performance Simulation Association (IBPSA) Conference. 
Berners-Lee, T., Hendler, J., and Lassila, O. (2001). "The semantic web." Sci. Am. 284, 35-43.

buildingSMART (2012). "IFC solutions factory, The model view definition site." http://www.blis-project.org/IAI-MVD/ (Nov. 26, 2013).

buildingSMART (2013). "BuildingSMART - International home of OpenBIM." http://buildingsmart.org/ (Nov. 26, 2013).

Eastman, C., Teicholz, P., Sacks, R., and Liston, K. (2011). BIM Handbook: A Guide to Building Information Modeling for Owners, Managers, Designers, Engineers and Contractors, Wiley, Hoboken, NJ.

ISO (2013). ISO 16739:2013: Industry Foundation Classes (IFC) for data sharing in the construction and facility management industries.

LBNL (2013). "Simergy: Simergy homepage." https://simergy-beta.lbl.gov/ (Nov. 26, 2013).

Liebich, T., Adachi, Y., Forester, J., Hyvarinen, J., Karstila, K., Reed, K., Richter, S., and Wix, J. (2009). "Industry Foundation Classes IFC2x edition 3 technical corrigendum 1." http://www.buildingsmart-tech.org/ specifications/ifc-releases/ifc2x3-tc1-release/summary (Nov. 15, 2013).

Manola, F., and Miller, E. (2004). "RDF Primer - W3C Recommendation 10 February 2004." http://www.w3.org/TR/rdf-primer/ (Nov. 15, 2013).

O’Donnell, J., Maile, T., Rose, C., Mrazovic, N., Morrissey, E., Parrish, K., Regnier, C., and Bazjanac, V. (2013). "Transforming BIM to BEM: Generation of building geometry for the NASA Ames sustainability base BIM." Technical Report No. LBNL-6033E, Lawrence Berkeley National Laboratory, Berkeley, CA.

O’Donnell, J.T., Richard See, Maile, T., Bazjanac, V., and Haves, P. (2011). "SimModel: A domain data model for whole building energy simulation." 12th International Building Performance Simulation Association (IBPSA) Conference.

Pauwels, P., De Meyer, R., and Van Campenhout, J. (2010). "Interoperability for the design and construction industry through semantic web technology." 5th International Conference on Semantic and Digital Media Technologies. in LNCS 6725, pp. 143-158.

Pauwels, P., Van Deursen, D., De Roo, J., Van Ackere, T., De Meyer, R., Van de Walle, R., and Van Campenhout, J. (2011). "Three-dimensional information exchange over the semantic web for the domain of architecture, engineering, and construction." Ai. Edam. 25, 317-332.

See, R., Haves, P., Sreekanthan, P., Basarkar, M., O’Donnell, J., and Settlemyre, K. (2011). "Development of a user interface for the EnergyPlus whole building energy simulation program." 12th International Building Performance Simulation Association (IBPSA) Conference.

Törmä, S. (2013). "Semantic linking of building information models." 7th IEEE International Conference on Semantic Computing.

W3C OWL Working Group (2012). "OWL 2 Web Ontology Language Document Overview (Second Edition) - W3C Recommendation 11 December 2012. " http://www.w3.org/TR/owl2-overview/ (Nov. 15, 2013). 